\title{
A 3-DIMENSIONAL FORCE FIELD METHOD FOR ROBOT COLLISION AVOIDANCE IN COMPLEX ENVIRONMENTS
}

\author{
P. Chotiprayanakul, D. K. Liu, D. Wang and G. Dissanayake \\ ARC Centre of Excellence for Autonomous Systems \\ Faculty of Engineering, University of Technology, Sydney \\ Broadway, NSW 2007, Australia \\ \{pchotipr, dkliu, Da-Long.Wang, gdissa\}@eng.uts.edu.au
}

\begin{abstract}
This paper proposes a three-dimensional force field (3D-F $\mathrm{F}^{2}$ method for efficient motion planning and collision avoidance of a $6 \mathrm{DOF}$ manipulator in complex and dynamic environments while keeping the planned end-effector's path and speed unchanged. The $3 \mathrm{D}-\mathrm{F}^{2}$ is defined as ellipsoid shapes covering selected links of a manipulator. When the manipulator moves and its ellipsoid force field approaches to an obstacle in a tolerant range, a repulsive force will be generated and considered in the robot kinematic and dynamic analyses. In infrastructure maintenance, spray-painting and sand-blasting operations require that the operating spot "moves" smoothly and continuously along planned path on a work surface at a constant speed, and allow changes in length and orientation of the spray/blasting stream. Thus, the stream is supposed to be another link and the end of stream performs as a spherical joint fixed on the target surface. Various simulations in a construction area show that the $3 \mathrm{D}-\mathrm{F}^{2}$ can retain the operating path and effectively avoid potential collisions.
\end{abstract}

\section{KEYWORDS}

Force Field, 3D-F², Collision Avoidance

\section{INTRODUCTION}

This paper addresses the motion planning and collision avoidance problem for a robot arm in complex environments. Path/motion planning includes off-line and real-time path planning that generates paths automatically for a robot arm to move from one position to another. When planned paths are executed by a robot arm, the robot control system needs to generate a collision-free trajectory and drive every joint of the robot safely and smoothly. Collision avoidance for a whole robot manipulator has been considered as a very important issue, especially in complex and dynamic environments. Distance measuring is a procedure, before collision avoidance, to determine the possibility of collisions. As computing the distance from a manipulator to obstacles using accurate topology is very time consuming, many researchers have been trying to find efficient approaches to speed up the calculation, for example, covering the links of a robot manipulator with polytope, polyhedron [5], [6], sphere [7], [8], [10], [11], ellipsoid, etc., which can significantly reduce computing time. Based on the measured distance between the robot arm and obstacles in the environment, collision avoidance algorithms are then used to steer the movement of a robot arm away from obstacles while fulfilling its task. Many techniques for this objective, for example the Configuration Space (C-Space) method [2], [3], potential field approach [4], [9] and force field method [11], [12], have been developed.

Path/motion planning and collision avoidance have been extensively investigated. Biegelbauer et al. generalised the painting path planning process [1], which is capable of generating collision-free trajectory for the end-effector. Lozano-Perez presented approaches to distance measuring 
between polyhedrons which are used for path planning in discrete C-space [2], [3]. Khatib applied the potential field concept for real-time obstacle avoidance for manipulators and mobile robots [4]. Juang applied polyhedrons to represent a manipulator and potential field method to dynamically control manipulators [5], [6]. Greenspan and Burtnyk modelled a robot arm and obstacles with sets of spheres, measured the distances with a weighted voxel map and demonstrated successful collision avoidance and on-line path planning [7], [8]. Lin and Chuang used potential fields in 3-D workspace to generate a collision free path by locally adjusting the robot configuration for minimum potential [9]. Brock and Khatib presented elastic strip framework generating an obstacle bended free space tunnel for a robot to move from point to point [10]. Xie et al. used spheres covering a robot and converted the repulsive forces to joint torques to find the collision free trajectory [11]. D.Wang et al. presented a variable speed force field $\left(\mathrm{VSF}^{2}\right)$ method in 2D for multi-mobile robot collaboration and collision avoidance [12]. A relationship between speed and repulsive force is defined to reduce the oscillation in robot speed and orientation.

In operations such spray-painting, the end-effecter of a manipulator is required to move at constant speed along pre-planned path while considering the collision avoidance of the whole robot arm. Collision avoidance for the whole body of a robot arm has not been extensively studied. We propose a $3 \mathrm{D}-\mathrm{F}^{2}$ method in this research. The definition of ellipsoidal three-dimensional Force Field (3D-F ${ }^{2}$ ) covering the links of a manipulator are introduced in Section 2. Section 3 presents a robot model and the approach using $3 \mathrm{D}-\mathrm{F}^{2}$ to fulfil a task. Simulations carried out in a maintenance environment shows that the $3 \mathrm{D}-\mathrm{F}^{2}$ effectively protects the manipulator from possible collisions with obstacles in a complex environment. The remarkable computing time shows that the $3 \mathrm{D}-\mathrm{F}^{2}$ method can be used in realtime applications.

\section{DEFINITION OF THE 3D-F ${ }^{2}$}

This section introduces the definition of the $3 \mathrm{D}-\mathrm{F}^{2}$. To determine the ellipsoid covering a link of manipulator, two points on a link are selected as the foci of the resulted ellipsoid $\left(\mathrm{P}_{1}\right.$ and $\mathrm{P}_{2}$ in Fig.
1). Transformations from the robot joint coordinate system to the global system are given by transfer function $\left({ }^{i} \mathbf{T}_{j}\right)$ and rotation-translation metrics $\left({ }^{i} \mathbf{A}_{j}\right)$. $\mathbf{P}_{i(u, v, w)}$ and $\mathbf{P}_{i(x, y, z)}$ are points on the robot's coordinate system and the global system, respectively.

$$
\begin{aligned}
& { }^{0} \mathbf{T}_{n^{t h}}=\prod_{m=0}^{n^{t h}-1}{ }^{m} \mathbf{A}_{m+1} \\
& \mathbf{P}_{i(x, y, z)}={ }^{0} \mathbf{T}_{n^{t h}} \mathbf{P}_{i(u, v, w)} \quad ; \text { where } i=(1,2)
\end{aligned}
$$
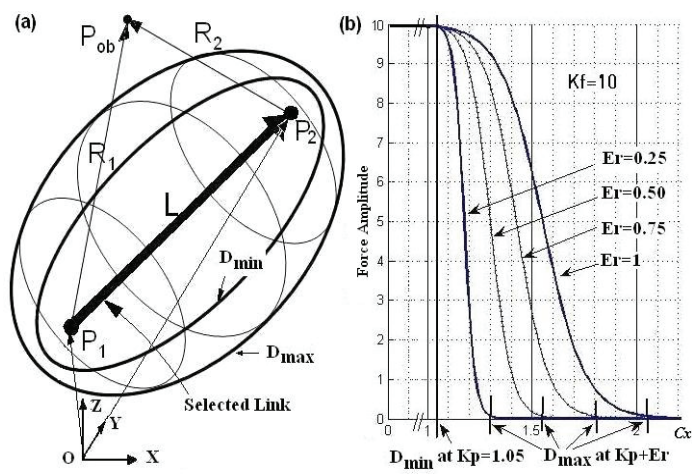

\section{Figure 1 (a) Factors of $3 D-F^{2}$ (b) Amplitude of Repulsive Force Between $D_{\min }$ and $D_{\max }$}

The equations of $D_{\min }$ and $D_{\max }$ ellipsoids are given in (3) and (4). To ensure that this $D_{\min }$ ellipsoid will cover the whole body of the link, the length of the major axis is set to be equal to $\mathrm{L}^{*} K_{p}$, where $\mathrm{L}$ is the distance between the foci and $K_{p}$ is a constant larger than 1 . For any point in the $3 \mathrm{D}$ space $\left(\mathrm{P}_{\mathrm{ob}}\right.$ in Fig. 1), if the sum of distances, $\mathrm{R}_{1}$ and $R_{2}$, between this point to the foci is equal to the length of the major axis, that is, $\mathrm{L}^{*} K_{p}$, this point is on the $\mathrm{D}_{\text {min }}$ ellipsoid. If $\mathrm{R}_{1}+\mathrm{R}_{2}$ is smaller than $\mathrm{L}^{*} K_{p}$, this point is inside $\mathrm{D}_{\text {min }}$. On the contrary, when $\mathrm{R}_{1}+\mathrm{R}_{2}$ is larger than $\mathrm{L}^{*} K_{p}$, the point is outside $D_{\text {min }}$ (see Fig. 1). To take the linear speed of the end-effecter into account, we introduce a new factor, $E_{r}$, which is the ratio of the instant speed $\left(V_{i}\right)$ and the maximum speed $\left(V_{\max }\right)$, so $E_{r}$ is between 0 to1. The length of major axis of $\mathrm{D}_{\text {max }}$ is set to be $\mathrm{L}^{*}\left(K_{p}+E_{r}\right)$.

$$
\begin{aligned}
& \mathbf{L} * K_{p}=\left|\overrightarrow{\mathbf{R}}_{1}\right|+\left|\overrightarrow{\mathbf{R}}_{2}\right| \\
& \mathbf{L} *\left(K_{p}+E r\right)=\left|\overrightarrow{\mathbf{R}}_{1}\right|+\left|\overrightarrow{\mathbf{R}}_{2}\right| \\
& E_{r}=\frac{V_{i}}{V_{\max }}
\end{aligned}
$$

From (3) and (4), if we define $C x$, is a ratio between $\mathbf{R}_{1}+\mathbf{R}_{2}$ and $\mathbf{L}$, the $K_{p}$ and $K_{p}+E r$ will 
indicate if $\mathbf{P}_{o b}$ is inside or between or outside the $\mathrm{D}_{\min }$ ellipsoid and the $\mathrm{D}_{\max }$ ellipsoid.

$C x=\frac{\left|\overrightarrow{\mathbf{R}}_{1}\right|+\left|\overrightarrow{\mathbf{R}}_{2}\right|}{|\mathbf{L}|}$

Then, the amplitude of repulsive force is given by

$$
\left|\overrightarrow{\mathbf{F}}_{r e p}\right|=K f-\left(\frac{K f}{1+e^{\left(-\frac{10(C x-K p-0.5 E r)}{E r}\right)}}\right)
$$

$K f$ is maximum repulsive force. Fig 1 b shows how the repulsive force varies with $E_{r}$ and the position between $D_{\min }$ and $D_{\max }$. This definition ensures the smooth change of the force. The direction of the repulsive force is defined to be the unit vector that points from $\mathbf{P}_{\mathrm{ob}}$ to $\mathbf{P}_{1}$ or $-\mathrm{R}_{1}$.

$\overrightarrow{\mathbf{F}}_{r e p}=\frac{\overrightarrow{\mathbf{P}}_{1}-\overrightarrow{\mathbf{P}}_{o b}}{\left|\overrightarrow{\mathbf{P}}_{1}-\overrightarrow{\mathbf{P}}_{o b}\right|} *\left|\overrightarrow{\mathbf{F}}_{r e p}\right|$

\section{KINEMATICS AND DYNAMICS}

In the section, we analysis the manipulator's kinematics and dynamics in operations such as spray-painting and then introduce how the $3 \mathrm{D}-\mathrm{F}^{2}$ method can be applied for planning and collision avoidance.

\subsection{Model of Robot}

In a spraying operation, the end effector of the manipulator needs to follow a pre-planned path and move continuously. To maintain the target point, the manipulator model has to be adapted by assuming a virtual link from the robot end-effecter to the target point and a virtual joint at the spraying spot which contains three rotating axes $\left(\mathrm{R}_{z, \psi} \mathrm{R}_{\mathrm{y}, \zeta} \mathrm{R}_{\mathrm{x}, \rho}\right)$. Thus, the model of the robot arm can be represented in Figure 2. This modified model has two fixed pivots at both ends of a serial chainlink. The stream pivot is an instant point that moves along a pre-planned path.

\subsection{The Effects of Repulsive Force}

When an obstacle or any part of an obstacle is inside of the $\mathrm{D}_{\max }$ ellipsoid of the virtual stream link, a repulsive force will be generated on a point that has the smallest $C x$ and has maximum repulsive force. Moment $\left(\overrightarrow{\mathbf{M}}_{1, \psi, \varsigma, \rho}\right)$, which is a cross product of the distance vector of stream direction $\left(\overrightarrow{\mathbf{l}}_{\text {stream link }}\right)$ and the force $\left(\overrightarrow{\mathbf{F}}_{1}\right)$, is defined by (11) and will change the stream direction. The repulsive force at the lower arm $\left(\overrightarrow{\mathbf{F}}_{2}\right)$ is defined from the nearest point of an obstacle and is toward to the end of lower arm at $\mathbf{P}_{1,2}$.

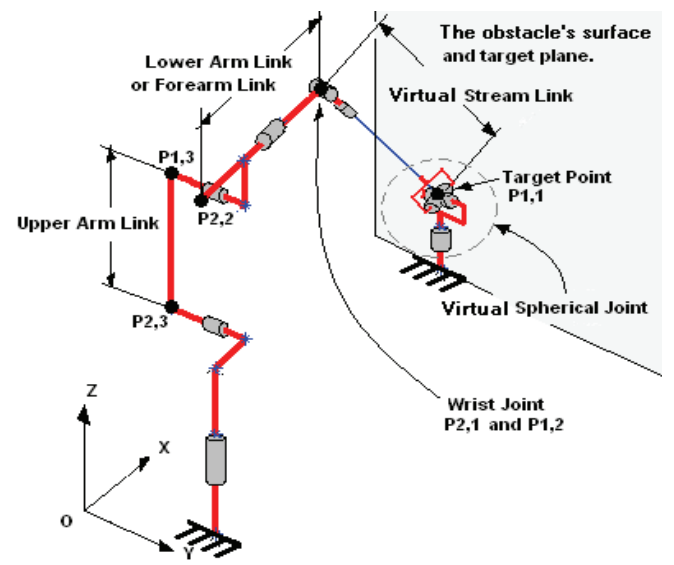

Figure 2 The Stream Link, Virtual Spherical Joint and Points of the Robot Arm

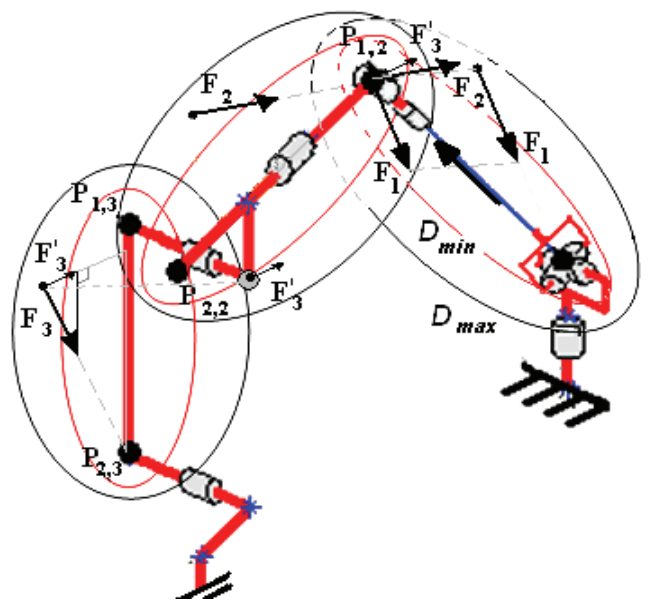

Figure 3 Repulsive Forces on the Links of a Robot Arm

$\overrightarrow{\mathbf{M}}_{1, \psi, \varsigma, \rho}=\left[\begin{array}{c}M_{1, \psi} \\ M_{1, \varsigma} \\ M_{1, \rho}\end{array}\right]=\overrightarrow{\mathbf{l}}_{\text {stream link }} \times \overrightarrow{\mathbf{F}}_{1}$

As shown in Figure 3, $\overrightarrow{\mathbf{F}}_{2}$ is going to change the stream link direction by pushing or pulling the wrist of the robot arm. In a different way, the repulsive force of the upper $\operatorname{arm}\left(\overrightarrow{\mathbf{F}}_{3}\right)$ is divided into two perpendicular vectors, one points to the upper arm link at $\mathrm{P}_{2,3}$ the shoulder joint and another $\left(\overrightarrow{\mathbf{F}}_{3}^{\prime}\right)$ to its perpendicular plane. The force would have 
an effect to the rotation of shoulder indirectly because it could make the robot missing the target. While the force $\overrightarrow{\mathrm{F}}_{3}^{\prime}$ is pushing the elbow of the robot, there is a reaction against the force. With this force, the forearm transfers the $\overrightarrow{\mathbf{F}}_{3}^{\prime}$ in parallel along itself to push the stream as well as the repulsive force from lower arm.

$$
\begin{aligned}
& \overrightarrow{\mathbf{M}}_{2, \psi, \varsigma, \rho}=\overrightarrow{\mathbf{l}}_{\text {stream link }} \times \overrightarrow{\mathbf{F}}_{2} \\
& \overrightarrow{\mathbf{M}}_{3, \psi, \varsigma, \rho}=\overrightarrow{\mathbf{l}}_{\text {streamlink }} \times \overrightarrow{\mathbf{F}}_{3}^{\prime} \\
& \overrightarrow{\mathbf{M}}_{r e p_{\psi, \varsigma, \rho}}=\sum_{i=1}^{3} \overrightarrow{\mathbf{M}}_{i, \psi, \varsigma, \rho}
\end{aligned}
$$

If there are no obstacles, repulsive forces become zero, the end-effecter of the robot end-effecter has to return to the planned orientation as the result of a virtual torque $\overrightarrow{\mathbf{M}}_{s p, \psi, \varsigma, \rho}$ defined by (13). $\quad \mathbf{T}_{\psi, \varsigma, \rho}$ and $\mathbf{A}_{\psi, \varsigma, \rho}$ are the planned orientation and the actual orientation of the end-effecter, respectively, and $K s p$ is a virtual spring constant.

$$
\overrightarrow{\mathbf{M}}_{s p, \psi, \varsigma, \rho}=K s p *\left(\mathbf{A}_{\psi, \varsigma, \rho}-\mathbf{T}_{\psi, \varsigma, \rho}\right)
$$

Thus the torques are applied to the robot arm will be given by

$$
\overrightarrow{\mathbf{M}}_{\psi, \varsigma, \rho}= \begin{cases}\overrightarrow{\mathbf{M}}_{r e p_{\psi, \varsigma, \rho}} & ; \text { one of } \mathbf{F}_{i, \text { rep }}>0 \\ \overrightarrow{\mathbf{M}}_{s p p_{\psi, \varsigma, \rho}} & ; \text { all of } \mathbf{F}_{i, \text { rep }}=0\end{cases}
$$

\subsection{Inverse Kinematics and Dynamics}

The dynamics of the stream link can be described with spatial motion equations (16-18). Parameters in the equations include target position $\left(\mathbf{S}_{\mathrm{t}}\right)$, start position $\left(\mathbf{S}_{\mathrm{s}}\right)$, current position $\left(\mathbf{S}_{\mathrm{c}}\right)$, previous position $\left(\mathbf{S}_{\mathrm{p}}\right)$, speed $\left(\mathrm{v}_{\mathrm{p}}\right)$, differential time $(\mathrm{dt})$, direction of the path $(\mathbf{D})$, current orientation $\left(\mathbf{O}_{\mathrm{c}}\right)$, previous orientation $\left(\mathbf{O}_{\mathrm{p}}\right)$ and the mass property of the virtual stream link $(\mathrm{Km})$.

$$
\begin{aligned}
& \mathbf{D}=\frac{\mathbf{S}_{\mathrm{t}}-\mathbf{S}_{\mathrm{s}}}{\left\|\mathbf{S}_{\mathrm{t}}-\mathbf{S}_{\mathrm{s}}\right\|} \\
& \mathbf{V}=\mathrm{v}_{\mathrm{p}} * \mathbf{D} \\
& \mathbf{S}_{\mathrm{c}}=\mathbf{S}_{\mathrm{p}}+\mathbf{V}^{*} \mathrm{dt} \\
& \mathbf{O}_{\mathrm{c}}=\mathbf{O}_{\mathrm{p}}+K m^{*} 0.5 * \overrightarrow{\mathbf{M}}_{\psi, \varsigma, \rho} * \mathrm{dt}^{2}
\end{aligned}
$$

The poses of the manipulator joints, $[\boldsymbol{\theta}]$, can be obtained from the position and orientation in the
Cartesian space $[\mathrm{x} \mathrm{y} \mathrm{z} \psi \zeta \rho]$ by applying inverse kinematics (IK):

$[\boldsymbol{\theta}]=\boldsymbol{I K}\left(\left[\begin{array}{ll}\mathbf{S}_{\mathrm{c}} & \mathbf{O}_{\mathrm{c}}\end{array}\right],\left[\boldsymbol{\Theta}_{\text {previous }}\right]\right)$

$\left[\boldsymbol{\Theta}_{\text {previous }}\right]$ is the joint poses in the previous step, which is used in pose selection to reduce jerk in joint movement. The angles of the first three joints, shoulder to elbow, come from the coordinate of the wrist joint $\left(\mathbf{P}_{2,1}\right.$ current $)$ from (20). Input the first three joints' angles, $\Theta_{1}, \Theta_{2}, \Theta_{3}$ to ${ }^{0} \mathbf{T}_{6}$ to work out ${ }^{3} \mathbf{T}_{6}$ in term of $\left[\mathbf{n}^{\prime} \mathbf{s}^{\prime} \mathbf{a}^{\prime}\right]$ in (23) and compare it with ${ }^{3} \mathbf{T}_{6}$ in (23) from forward function to get $\Theta_{4}, \Theta_{5}, \Theta_{6}$.

$$
\left[\begin{array}{cc}
\mathbf{P}_{2,1} \text { current } \\
1
\end{array}\right]=\left[\begin{array}{cc}
\mathbf{R}_{z, O(\psi)} & \mathbf{0} \\
\mathbf{0} & 1
\end{array}\right]\left[\begin{array}{cc}
\mathbf{R}_{y, O(S)} & \mathbf{0} \\
\mathbf{0} & 1
\end{array}\right]\left[\begin{array}{cc}
\mathbf{R}_{x, O(\rho)} & \mathbf{0} \\
\mathbf{0} & 1
\end{array}\right]\left[\begin{array}{c}
0 \\
0 \\
-l_{\text {streamlink }} \\
1
\end{array}\right]+\left[\begin{array}{c}
S C \\
1
\end{array}\right]
$$

${ }^{0} \mathbf{T}_{3}{ }^{3} \mathbf{T}_{6}={ }^{0} \mathbf{T}_{6}=\mathbf{R}_{z, O c(\psi)} \mathbf{R}_{y, O c(\zeta)} \mathbf{R}_{x, O c(\rho)}=\left[\begin{array}{ccc}n_{x} & s_{x} & a_{x} \\ n_{y} & s_{y} & a_{y} \\ n_{z} & s_{z} & a_{z}\end{array}\right]$

${ }^{3} \mathbf{T}_{6}=\frac{{ }^{0} \mathbf{T}_{3}^{T}}{\left|{ }^{0} \mathbf{T}_{3}\right|}\left[\begin{array}{ccc}n_{x} & s_{x} & a_{x} \\ n_{y} & s_{y} & a_{y} \\ n_{z} & s_{z} & a_{z}\end{array}\right]=\left[\begin{array}{ccc}n_{x}^{\prime} & s_{x}^{\prime} & a_{x}^{\prime} \\ n_{y}^{\prime} & s_{y}^{\prime} & a_{y}^{\prime} \\ n_{z}^{\prime} & s_{z}^{\prime} & a_{z}^{\prime}\end{array}\right]$

${ }^{3} \mathbf{T}_{6}={ }^{3} \mathbf{A}_{4}{ }^{4} \mathbf{A}_{5}{ }^{5} \mathbf{A}_{6}$

\section{SIMULATION AND RESULTS}

Simulations are carried out in an infrastructure maintenance environment. A manipulator mounted on a lift platform is supposed to spray-paint certain area of a maintenance site. The manipulator model in these simulations is Denso 6556 [13]. The simulation program runs on MATLAB7.1 in a Pentium 4 computer with a $2.8 \mathrm{GHz}$ CPU and $1 \mathrm{~GB}$ RAM. The values of parameters in the simulations are set to be $\mathrm{v}_{\mathrm{p}}=30 \mathrm{~mm} / \mathrm{sec}, d t=0.1 \mathrm{sec}, K m=2$, $K_{f}=\left[\begin{array}{lll}50 & 20 & 20\end{array}\right], K_{p}=\left[\begin{array}{lll}1.01 & 1.1 & 1.1\end{array}\right], \quad E r=\left[\begin{array}{lll}0.025 & 0.15\end{array}\right.$ $0.15]$ and $K s p=2$.

\subsection{Case 1}

The robot is lifted under a ceiling and spray-paints the surface of the ceiling. Figure 4 shows simulation snapshots of the robot arm with $3 \mathrm{D}$ force field, $\mathrm{D}_{\min }, \mathrm{D}_{\max }$, and the end effector's paths. Points 1 to 4 are a pre-planned path denoted by the solid blue line in Fig. 4a. The spray-painting direction is set to be normal to the ceiling surface 
in this task. The robot performs a motion that avoids crashing into a flank on the wall and a hanging ventilator beside it. Figure 5 shows the changes of joint angles from point 1 to 4 in Fig 4 a. The end-effecter of the manipulator traveled 4600 $\mathrm{mm}$ in total in 153 seconds. The computation time required in this simulation is only 63 seconds.

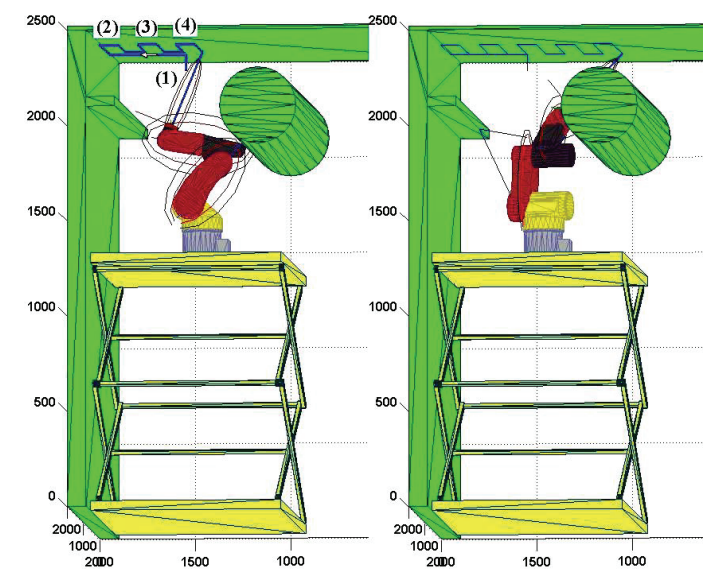

Figure 4 Case1: Ceiling-Spraying Task

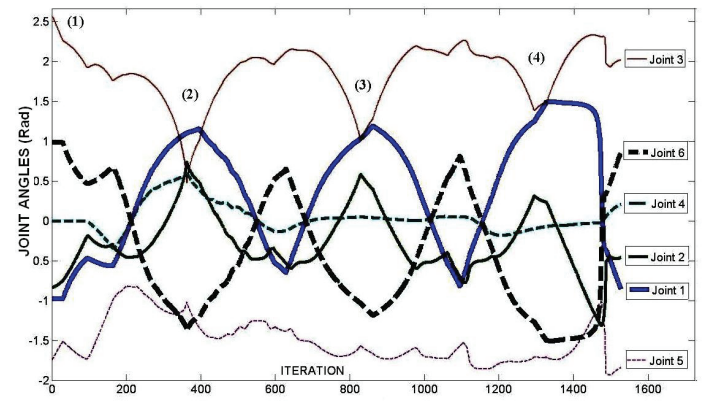

Figure 5 Angles of Joints Along the Path (Case 1)

\subsection{Case 2}

In this simulation, a vertical wall is painted by the robot (Fig. 6) and the resulted joints' angles are shown in Fig. 7. The path is set upon a vertical side of the wall. The simulation is carried out using the same end-effecter speed and the same force field parameters as those in Case 1, except that stream length is set as $400 \mathrm{~mm}$ the end-effecter of the manipulator traveled $2750 \mathrm{~mm}$ in total in 92 seconds and the computation time required is 46 seconds only.

\subsection{Case 3}

The robot paints a hanging ventilator (Fig. 9) and joints' angles are shown in Fig. 10. The path is set upon the surface of the cylindrical ventilator. The simulation is carried out using the same endeffecter speed and the same force field parameters as those in Case 1, except that stream length is set as $350 \mathrm{~mm}$ the end-effecter of manipulator traveled $2300 \mathrm{~mm}$ in 77 seconds and the computation time required is 32 seconds.

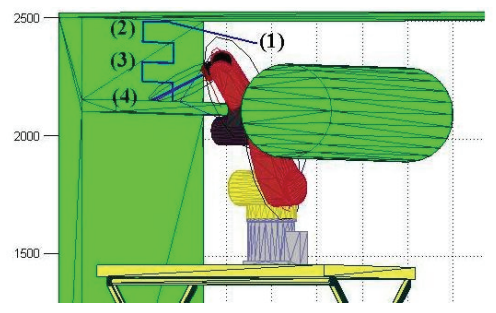

Figure 6 Case2: Wall Spraying Task

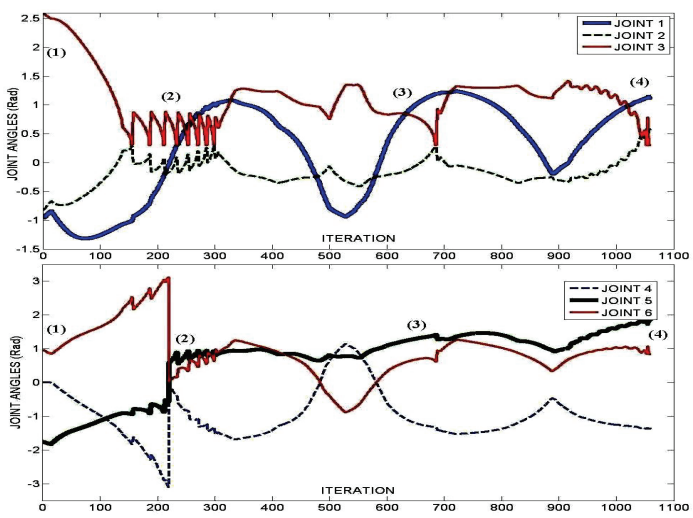

Figure 7 Angles of Joints Along the Path (Case 2)

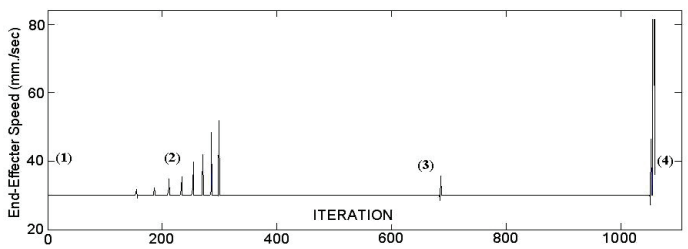

Figure 8 End-Effecter Speed in Case2

\subsection{Discussion}

The simulation results show that the $3 \mathrm{D}-\mathrm{F}^{2}$ method is able to prevent the robot arm from collisions with obstacles in a complex environment. It also can be seen clearly that the $3 \mathrm{D}-\mathrm{F}^{2}$ method is fast enough to be implemented in the real-time trajectory planning and collision avoidance for manipulators. It can be seen from Fig. 7 and Fig. 10 that the angle changes of some joints are not always smooth although the path is a straight line. Oscillations occur when the robot arm reaches to 
some corner areas, such as point 2 in Fig. 6 and point 4 in Fig. 9. In the case of Fig 6, the force field of the stream needs to protect the stream link from obstacles from several directions, i.e. ceiling and wall. The stream link will suffer the repulsive forces from ceiling and wall alternatively, which causes the rapid change of the orientation of stream link and the linear speed of stream link on the working surface (see Fig. 8 and Fig. 11). As a result, the spraying point will fluctuate around the target point. Please note that these fluctuations are instantaneous and only 1 or 2 iterations or 0.1 to 0.2 seconds. They can be easily reduced by applying a damper factor. Besides, there are a few situations that the robot arm reaches its joint limit or workspace limit. These problems will be addressed in our future research work.

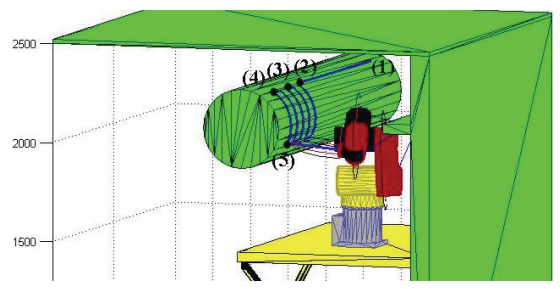

Figure 9 Case3: Ventilator Spraying Task

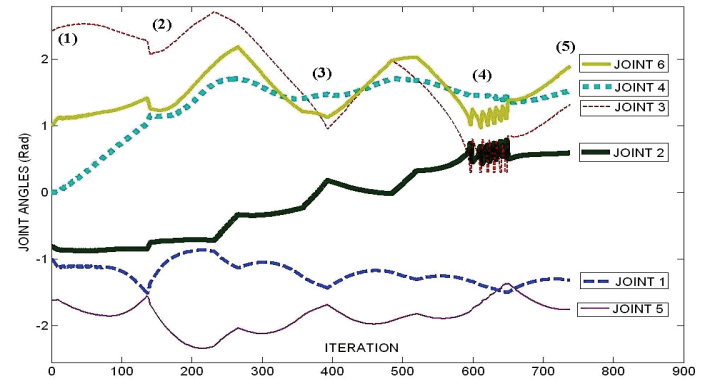

Figure 10 Angles of Joints Along Path (Case3)

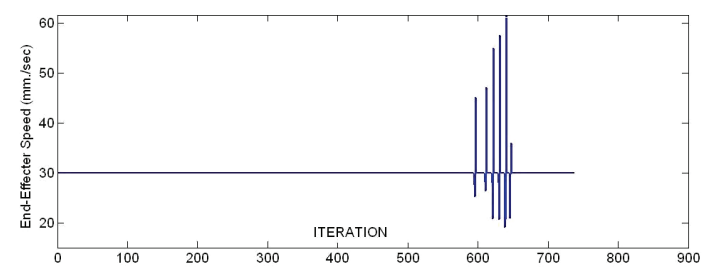

Figure 11 End-Effecter Speed of Case3

\section{CONCLUSION AND FUTURE WORK}

This paper presented a novel $3 \mathrm{D}-\mathrm{F}^{2}$ method for real-time collision avoidance and motion planning of manipulators in known complex and dynamic environments. With the $3 \mathrm{D}-\mathrm{F}^{2}$, a manipulator is capable of moving its end-effector along a preplanned path in a constant speed while avoiding obstacles simultaneously. Simulations carried out in a complex environment demonstrate the efficiency and feasibility of the $3 \mathrm{D}-\mathrm{F}^{2}$. In a spray operation, stream length is allowed to change in a certain range, which introduces another degree of freedom. In the next stage of research, the stream link will be represented with a prismatic joint. Further experiments with a real robot in a real working environment will also be conducted.

\section{ACKNOWLEDGMENT}

This work is supported in part by the ARC Centre of Excellence for Autonomous System, funded by Australian Research Council (ARC) and the New South Wales State Government, Australia.

\section{REFERENCES}

[1] G. Biegelbauer, A. Pichler, M. Vincze, C. Nielsen, H. J. Andersen and K. Haeusler (2005) Automatic Generation of Robot Painting Motions for Unknown Parts, IEEE Robotics \& Automation Magazine, 1070-9932,pp.24-34.

[2] T. Lozano-Perez (1983) Spatial Planning: A Configuration Space Approach, IEEE Transactions on Computers, vol. C-32, no.2, pp.108-119.

[3] T. Lozano-Perez (1987) A Simple MotionPlanning Algorithm for General Robot Manipulators, IEEE Journal of Robotics and Automation, vol. RA-3, no.3, pp. 224-238.

[4] O. Khatib (1985) Real-Time Obstacle Avoidance for Manipulators and Mobile Robots, IEEE Int. Conf. on Robotics and Automation, pp.500-505.

[5] J.-G. Juang (1998) Collision Avoidance using Potential Fields ,Int. J. of Industrial Robot, MCB Uni Press, vol. 25,pp.408-415.

[6] J.-G. Juang (2004) Application of Repulsive Force and Genetic Algorithm to Multimanipulator Collision Avoidance Proceeding of $5^{\text {th }}$ Asian Conference, vol 2, pp.971-976.

[7] M. Greenspan and N. Burtnyk (1996) Obstacle Count Independent Real-Time Collision Avoidance, IEEE Int. Conf. on Robotics and Automation, Minnesota, pp.1073-1080. 
[8] M. Greenspan and N. Burtnyk (1994) Real Time Collision Detection, US Patent, Patent Number 5,347,459.

[9] C.-C. Lin and J. Chuang (2003) Potential-Based Path Planning for Robot Manipulators in 3-D Workspace, Int. Conf. on Robotics Automation, Taiwan, pp.3353-3358.

[10] O. Brock and O. Khatib (1999) Real-Time Obstacle Avoidance and Motion Coordination in a Multi-Robot Workcell, IEEE International Symposium on Assembly and Task Planning, Porto, Portugal, pp. 274-279.
[11]H. P. Xie, R. V. Patel, S. Kalaycioglu, H. Asmer (1998) Real-Time Collision Avoidance for Redundant Manipulator in an Unstructured Environment, IEEE Int. Conf. on Intelligent Robots and Systems, Victoria, B.C, Canada.

[12] D. Wang, D. Liu, G. Dissanayake (2006) A Variable Speed Force Field Method for Multirobot Collaboration, Int. Conf. on Intelligent Robots and Systems, Beijing, China.

[13] http://www.denso-wave.com/en/robot/ 